\title{
Solid-state nanopore easy chip integration in a cheap and reusable microfluidic device for ion transport and polymer conformation sensing
}

\author{
Jean Roman,,$+\downarrow$ Olivier Français, ${ }^{\uparrow}$ Nathalie Jarroux,${ }^{\ddagger}$ Gilles Patriarche,,$\S$ \\ Juan Pelta, ${ }^{,, \ddagger}$ Laurent Bacri, ${ }^{*, \ddagger}$ and Bruno Le Pioufle ${ }^{*, \dagger}$ \\ $\dagger$ †NS Paris-Saclay, CNRS, Institut d'Alembert, SATIE, Université Paris-Saclay, Cachan F-94230, \\ France \\ $\ddagger L A M B E$, Université Evry, CNRS, CEA, Université Paris-Saclay, Evry F-91025, France \\ \ESIEE-Paris, ESYCOM, Université Paris Est, Noisy-Le-Grand F-93160, France \\ $\S C 2 N$, CNRS, Université Paris-Sud, Université Paris-Saclay, C2N-Marcoussis, Marcoussis \\ F-91460, France \\ E-mail: juan.pelta@univ-evry.fr; laurent.bacri@univ-evry.fr; \\ bruno.le-pioufle@ens-cachan.fr
}

Solid-state nanopores have a huge potential in upcoming societal challenging applications in biotechnologies, environment, health and energy. Nowadays, these sensors are often used within bulky fluidic devices that can cause crosscontaminations and risky nanopore chips manipulations, leading to a short experimental lifetime. We describe the easy, fast and cheap innovative 3D-printer-helped protocol to manufacture a microfluidic device permitting the reversible integration of a silicon based chip containing a single nanopore. We show the relevance of the shape of the obtained channels thanks to finite elements simulations. We use this device to thoroughly investigate the ionic transport through the solid-state nanopore as a function of applied voltage, salt nature and concentration. Furthermore, its reliability is proved through the characterization of a polymer-based model of protein-urea interactions at the nanometric scale thanks to a hairy nanopore. KEYWORDS: microfluidics, solid-state nanopore, 3D-printing, ionic transport, protein-urea interactions, grafted polymer conformation, hydrodynamic simulations

Resistive pulse sensing is a rapidly growing method for macromolecules sequencing, nanopar- ticle detection and analysis ${ }^{1,2}$ or diagnosis ${ }^{3,4}$ based on the principle of the Coulter counter. ${ }^{5}$ An aperture made in an insulating material is placed between two voltage biased electrolytes. The ionic conductance of this aperture transiently decreases as a single nanoparticle passes through it. This electrical technique is used to characterize its size, ${ }^{6}$ conformation $^{7}$ or interactions with other particles ${ }^{8}$ at the single molecule scale. Two major resistive pulse sensing technique families can be considered: nanochannels and nanopores. Nanochannels feature a small aperture in regard of their length and consist of nanocapillaries, ${ }^{9}$ molded nanochannels ${ }^{10-12}$ and track-etched channels. ${ }^{13-16}$ The micrometric length of these nanochannels makes them less sensitive to particle translocation, for the conductance drop ratio is equal to the ratio between the volume of the empty channel and the one with the particle inside. In contrast, nanopores have similar nanometric length and diameter. They consist of either synthesized ${ }^{17-20}$ or proteic ${ }^{21,22}$ channels inserted in a lipid bilayer, or of a solid-state nanopore pierced in an ultra-thin dielectric membrane. ${ }^{8,12,23-30}$ The main advantage of protein channels is the reproducibility of their structure and their sensitiv- 
ity. ${ }^{31,32}$ However, theses pores are limited by the mechanical fragility of lipid bilayers and of their internal diameter, bound to a few nanometers.

In this work, we propose biomimetic nanopores made in solid membranes using techniques of the micro-electronic industry. ${ }^{23,30}$ These membranes are resistant to chemical, mechanical and heat stress. They are mainly pierced thanks to a focalized ion beam, ${ }^{23,24,27,33,34}$ an electron beam ${ }^{25,28,30}$ or more recently via dielectric breakdown ${ }^{35-37}$ to obtain a nanopore that matches the analyte size. Unfortunately, these membranes often bear a high surface energy. Thus, they are prone to particle or protein adsorption. ${ }^{27,38}$ Chemical, ${ }^{39}$ protein ${ }^{40,41}$ and polymer grafting $26,42,43$ or the use of $\mathrm{HfO}_{2}$ membranes ${ }^{29}$ has been proved to avoid these adsorptions and to increase nanopore conductance stability over time. In this study, we choose to graft polymer chains on the membrane.

Traditional and commercially available integrating devices are typically composed of two compartments open to the air and separated by the nanopore chip (Figure S1). Toric joints are often used to prevent any current leaking. Changing the analytes in such systems can only be done by emptying completely each compartment and then filling them up with the new analyte. Thus, crosscontamination between the analytes can only be avoided by reiterating the analyte change many times. The aperture to the nanopore chip and the joints hold a dead volume hardly reachable that increase the cross-contamination risk. In this publication, we propose to insert the nanopore chip into a microfluidic device. ${ }^{44,45}$ Based on micrometric channels directly in contact with the nanopore chip, this technology allows for rapid and efficient changes of analytes without dead volumes. Furthermore theses microfluidic channels hold only about $100 \mu \mathrm{L}$ of analytes. Whereas classical microfluidic nanopore-integrating devices are irreversibly sealed onto the nanopore chip, we show an easy way to obtain a reversible set-up, permitting to secure the valuable nanopore in between experiments.

The main difficulty of the making of PDMSbased microfluidic systems resides in the fabrication of a master mold. This is usually done using photolithography, ${ }^{46}$ a time-consuming technique requiring a clean room. Here, we propose a new way to rapidly produce low-cost microfluidic masters thanks to a $3 \mathrm{D}$-printer. ${ }^{47,48}$ Thanks to finiteelements simulations we show that the shape of the channels we obtain are better suited for the analysis of biological samples than typical ones as they yield a lesser hydrodynamic resistance and a more homogeneous shear stress.

In this work, we describe a new method to make an easy to use, unsealed device. It permits to use the same device with several nanopore chips and thus different pores or pore grafting or to reuse nanopore chips on different devices. We can also rapidly change the analyte without disassembling the nanopore chip from the microfluidic system.

We use this device with a nanopore grafted with long polymer chains. ${ }^{49}$ We discuss the nanopore conductance in a large range of $\mathrm{LiCl}$ and $\mathrm{KCl}$ salt concentrations in terms of ions and counter-ions transports. ${ }^{1,16,50,51} \mathrm{We}$ discuss the conformation of the polymer chain grafted on the $\mathrm{Si}_{3} \mathrm{~N}_{4}$ surface from the evaluation of apparent radius and length of the nanopore after polymer grafting.

As a proof of concept, the device is used for the analysis of grafted long chains conformations depending on the salt used. The electrical response of the pore leads us to a simple experimental proof for an important question about the mechanism of protein unfolding induced by urea. ${ }^{52-54}$

The design of the mold was performed using Blender (Blender foundation), a 3D graphics software. Instructions to the 3D-printer were then generated using the slicer Cura (Ultimaker B.V., Netherlands). We use an Ultimaker 2 3D-printer (Ultimaker B.V., Netherlands) to quickly fabricate the low-cost master for the molding of the PDMS sub-units of the device. This kind of printers is called Fused Deposition Modeling printers (FDM). It allows for the 2D patterning of melted material on a surface, leading to the fabrication, layer after layer, of a 3D object. In our case, only a few layers are necessary, each layer being $100 \mu \mathrm{m}$ thick. FDM printers can be used on any flat surface provided that the first layer of deposited material can stick to the substrate. We print the Poly(Lactic Acid) (PLA) master on a silicon wafer to benefit from its low roughness. We print at high temperature $\left(200^{\circ} \mathrm{C}\right)$ and at a low speed $(10 \mathrm{~mm} / \mathrm{s})$ to avoid residual stress inside the printed material and optimize print adhesion. Additional modifica- 
a) PDMS molding/spin coating (10min)

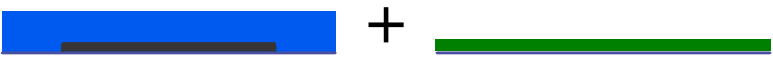

b) PDMS curing ( $1 \mathrm{~h} 30)$

c) Punching accesses $+1^{\text {st }}$ Plasma bonding (25min)

$2 \mathrm{x}$

d) Masking half of each pieces (1min)

$2 x$

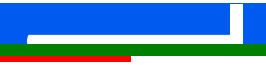

e) $2^{\text {nd }}$ Plasma bonding (20min)

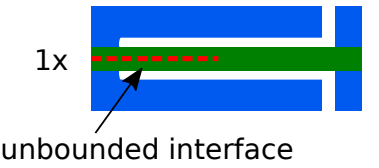

f) Inlet/outlet tubes insersion and PDMS casting (1h50)
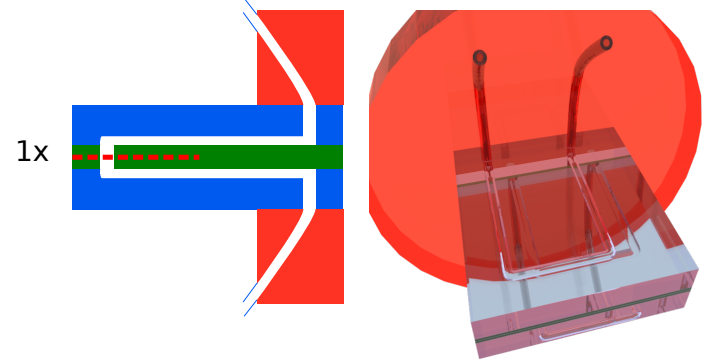

Figure 1: Protocol for the realization of the nanopore chip microfluidic device (not to scale). a) (left) The printed mold is covered by PDMS. (right) A thin PDMS film is produced by spin coating b) Curing at $75^{\circ} \mathrm{C}$. c) Plasma bonding (first) d) Masking e) Plasma bonding (second) f) Tube insertion and PDMS casting tions to the instructions code were made thanks to a custom program of our own. During the print, the substrate is heated up to $80^{\circ} \mathrm{C}$ to enhance print adhesion and limit the warping of printed objects due to the high temperature difference between the bottom of the object and the nozzle. Lastly, the freshly printed mold is heated up to $180^{\circ} \mathrm{C}$ for a few seconds using a hot plate. This last step is crucial in order to i) smooth the shape of the printed pattern and ii) achieve a good adherence of the PLA on the silicon wafer. Printed patterns were realized down to a width of $50 \mu \mathrm{m}$. However we chose to work with $150 \mu \mathrm{m}$ wide channels as it induces less hydrodynamic resistance and make for more robust masters. The three inches master contains eight of those channels, for the mounting of a total of four devices (Figure $2 \mathrm{a}$ ).

PDMS is used because of its insulating properties as well as its fluidic sealing properties. We use Sylgard 184 (Dow Corning, U.S.A.) with a 10:1 base to curing agent ratio. After a thorough mixing, the solution is degassed at 300 mbar for $10-15 \mathrm{~min}$. The fabrication steps of the microfluidic device we propose is detailed in Figure 1. The device is made of two identical PDMS subunits corresponding to the cis and trans sides of the nanopore chip. The latter will be inserted in between these sub-units (Figure 1a and c). Each sub-unit is composed of two parts: one main part containing the channel and the other one a thin film for closing the channel. The first one is cast on a 3D-printed master (Figure 1a), the channels dimensions are reported on Figure 2c. This layer should be around $5 \mathrm{~mm}$ high to be flexible enough to easily mount the nanopore chips without breaking and still be durable. Fluidic access to the channels are made at this step using a biopsy puncher. The thin film is spin-coated down to $200 \mu \mathrm{m}$ over a naked silicon wafer (Figure 1a). PDMS is cured at $75^{\circ} \mathrm{C}$ for $1 \mathrm{~h} 30$ (Figure 2b). Using a Basic Plasma Cleaner (Harrick Plasma, U.S.A.), the PDMS film is covalently bonded to the main part containing the channel to finalize the fabrication of each subunit (Figure 1c).

These sub-units are then partially bonded together. First, the top half of both these parts is masked with tape (red line represented in Figure 1d). Then, a second plasma activation bonds the unmasked bottom parts of the two sub-units. 

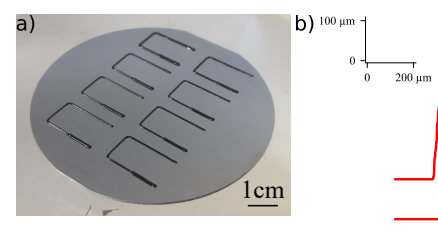

c) $\mathrm{PDMS}$ PDMS
Cischannel - Trans channe Fluidic access Electrodes

NanoporeChip $500 \mu \mathrm{m}$ -

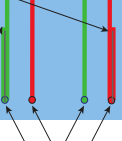

Fluidic accesses

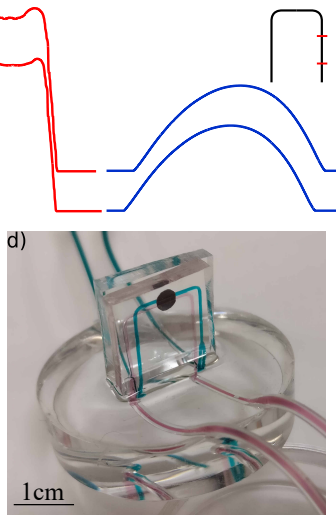

Figure 2: PDMS based device for easy solid-state nanopore chip use. a) Picture of mold 3D-printed on a 3 in silicon wafer. Four devices can be assembled from the eight printed patterns. b) Profiles of a 3D-printed mold before (red) and after melting. Two profiles are presented to show the uniformity of the printed parts, their location are approximately represented by red marks on the inset. c) Side and top schematic view of the device. d) Picture of the device with a mounted nanopore chip.

The upper part (dotted red line in Figure 1e) remains open for nanopore chip insertion. Fluidic access tubes are inserted in the holes we previously pierced. A talon of PDMS is cast to perfect their sealing (colored in orange in Figure 1f). For this, the device is put in a 2 in Petri dish which is then filled with PDMS. This talon also permits the device to stand for easy manipulations. Finally, using a biopsy puncher again, apertures are made through the thin layer covering the channel in place of where the nanopore chip will be inserted (Figure 2d). It will permit the analytes to reach the nanopore. To avoid any leaking during the changes of analytes, light pressure is applied to the upper part of the device thanks to two glass slates and a paper clip after nanopore mounting. This step is crucial to reversibly seal/detach the nanopore chips into/from the microfluidic device.

We use $3 \mathrm{~mm}$ silicon nitride chips (Nanopore solutions, Portugal) : these chips are composed of a $200 \mu \mathrm{m}$ thick silicon substrate, covered by a $20 \mathrm{~nm}$-thin $\mathrm{Si}_{3} \mathrm{~N}_{4}$ layer. The substrate is etched in its center to obtain a $15 \times 15 \mu \mathrm{m}^{2}$ free-standing $\mathrm{Si}_{3} \mathrm{~N}_{4}$ membrane (Figure 3a).

Nanopores are all pierced thanks to a Titan Themis TEM mode at $8 \mathrm{nA}$. The size of the probe is adapted to the desired pore size to obtain straight walls and a more stable pore. Thanks to the high currents used and the great control over the size of TEM beams, pores from 3 to $200 \mathrm{~nm}$ can be obtained rapidly. ${ }^{30}$ Immediately after piercing, the electron beam is defocused and we obtain an image of the pore. In this publication we use a $31 \mathrm{~nm}$ $\mathrm{Si}_{3} \mathrm{~N}_{4}$ pore (Figure 3 ).

PEG-7kDa (PolyPure AS, Norway) was grafted over the surface of the nanopore chip thanks to a three steps protocol already described in a previous paper ${ }^{42,55}$ and is briefly reported in the Supporting Information.

We use $\mathrm{Ag} / \mathrm{AgCl}$ electrodes directly inserted inside the channels of the device to measure the ionic current going through the pore. Current amplification was done thanks to an Axopatch 200B (Molecular devices, U.S.A.). Data was acquired with a PCle-6251 board (National Instruments, U.S.A.) interfaced with a custom .Net based program.

Current versus voltage curves (IVs) were obtained applying $30 \mathrm{~s}$ long voltage steps of $25 \mathrm{mV}$ between $\pm 200 \mathrm{mV}$. The current was recorded at $10^{3}$ samples/s and filtered thanks to the Axopatch 200B with a Bessel four poles low pass filter at $5 \mathrm{kHz}$. The average current for each voltage step is obtained from the last $15 \mathrm{~s}$ of the steps to avoid the expected capacitive surges induced by the changes of voltage. Finally, the slope of the IV curve yields the conductance of the pore according to the Ohm's law. Typical recordings as well as the resulting IV curves are shown in Figure 4a and b.

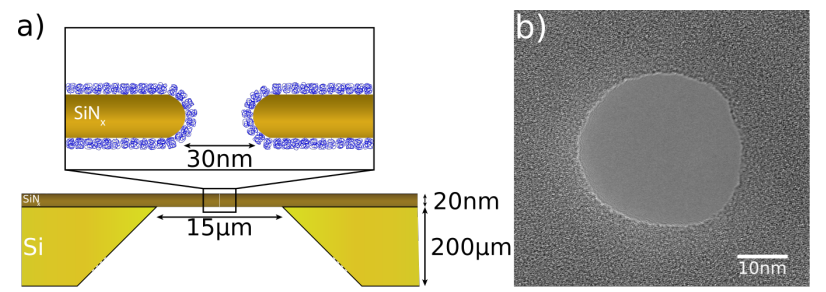

Figure 3: Morphology of a nanopore chip. a) Stylized side-view of a nanopore chip grafted with PEG-7kDa. b) TEM view of the pierced pore before grafting.

A schematics of a typical microfluidic system for solid-state nanopore measurements is shown in Figure S1. These systems are composed of two large compartments containing the electrolytes 
separated by the nanopore chip. The main drawback of such a system is that the change of analytes is done without a managed fluidic flow. The operator needs to change the electrolyte many times to avoid cross-contamination. Also, these systems often contain unreachable volumes of electrolytes, namely dead volumes, which accentuate this disadvantage. The use of a grazing channel facilitates the change of electrolytes and ensures less crosscontamination. These channels are a typical solution employed in microfluidics.

Previous approaches of printing microfluidic chips $^{48,56}$ or molds ${ }^{47}$ with FDM printers show that the spatial resolution is limited to $200 \pm 100 \mu \mathrm{m}$ depending on the 3D-printer used, with a roughness of $11 \mu \mathrm{m} .{ }^{48}$ To rise up to this challenge, we chose to print the mold over a silicon wafer to take advantage of the wafer nanometric smoothness. After printing, we melt the mold using a hot plate to enhance adhesion between the printed PLA and the silicon wafer. The profile of the 3D-printed mold has been measured thanks to a Dektak profilometer (Bruker, Germany) and is reported in Figure $2 b$. The height and width of the channel are constant along a straight part with $213 \pm$ $0.1 \mu \mathrm{m}$ height and $826 \pm 1 \mu \mathrm{m}$ width measured on both profiles. Also we measured the roughness of the printed parts of the mold. We show that the heating step allows to decrease the channel roughness from $R_{a}^{\text {raw }}=0.21 \pm 0.1 \mu \mathrm{m}$ and $R_{q}^{\text {raw }}=0.25 \pm 0.1 \mu \mathrm{m}$ to $R_{a}^{\text {melted }}=8 \pm 4 \mathrm{~nm}$ and $R_{q}^{\text {melted }}=10 \pm 4 \mathrm{~nm}$ (calculus explained in Supporting Information, Eq. 1). This low roughness allows for more durable molds as PDMS won't clutch to it when removed.

The reversible sealing of the device has been tested thanks to a simple manometer. The device withstands to up to 2 bar without leaking. Most of the time, old devices or intensively used ones only leak from the entry point of the access tubes. Thus, measurements are still possible with small leakage.

This microfluidic device permitted to record every set of data used for a graph within 7 hours each. The study we describe in the next section has been done along 4 weeks, dismounting the same nanopore chip at the end of the day to safely store it between experiments. This shows the reliability of the device for the handling of the fragile chip.
Whereas obtaining high channels is costly and difficult with traditional photolithography methods, our method allows for the fabrication of channels up to $1 \mathrm{~mm}$-high without hassle. An advantage of using high channels is their reduced hydrodynamic resistance. Thanks to this, the filling of the microfluidic system and the change of analytes in this device takes only 6 to $10 \mathrm{~s}$ for $1 \mathrm{~mL}$ injections by hand using a syringe. Also, we studied the effect of hemicylindrical channels on the fluidic flow using COMSOL Multiphysics finite elements simulations (COMSOL Group, Sweden). The flow speed and shear stress calculated for hemicylindrical, rectangular and cylindrical channels having the same cross-section area are reported in Figure S2. These simulations show the hemicylindrical channel yields a hydrodynamic resistance $3 \%$ lower than the rectangular one for a straight channel. Furthermore, the shear stress is more homogeneous along the wall in the hemicylindrical channel. Such homogeneity, especially along the walls of the channel is advantageous in tissue engineering, which is quite understandable as it corresponds to the shape of vascular vessels. A cylindrical channel allows for an even more homogeneous shear stress and a lesser hydrodynamic resistance. An assembly of two hemicylindrical channels obtained thanks to a 3D-printed mold could achieve such a channel. However, in our case, hemicylindrical channels are more suited to the integration of a nanopore chip.

Grafting long polymer chains on nanopores tends to reduce their apparent size. ${ }^{26}$ Theses chains presumably occupy space inside the pore and partially block the ion flux. The conformations of those chains inside or near the pore should greatly depend on their affinity with the electrolyte..$^{57} \mathrm{We}$ studied a $\mathrm{Si}_{3} \mathrm{~N}_{4} 31 \mathrm{~nm}$ pore grafted with a long polymer: PEG-7kDa. Those pores have been characterized intensely through the study of their conductance in $\mathrm{KCl}$ and $\mathrm{LiCl}$ buffers at various salt concentrations (Figure 4). First, we plot the current traces at fixed voltages between $-200 m V$ and $+200 m V$ (Figure 4a). In figure $4 \mathrm{a}$, we observe long fluctuations of the current in $10 \mathrm{mM} \mathrm{LiCl}$. This behavior is discussed in the Supporting Information.

From the average value of the current trace, we plot the rectification parameter II $(+200 \mathrm{mV}) / \mathrm{I}(-$ 

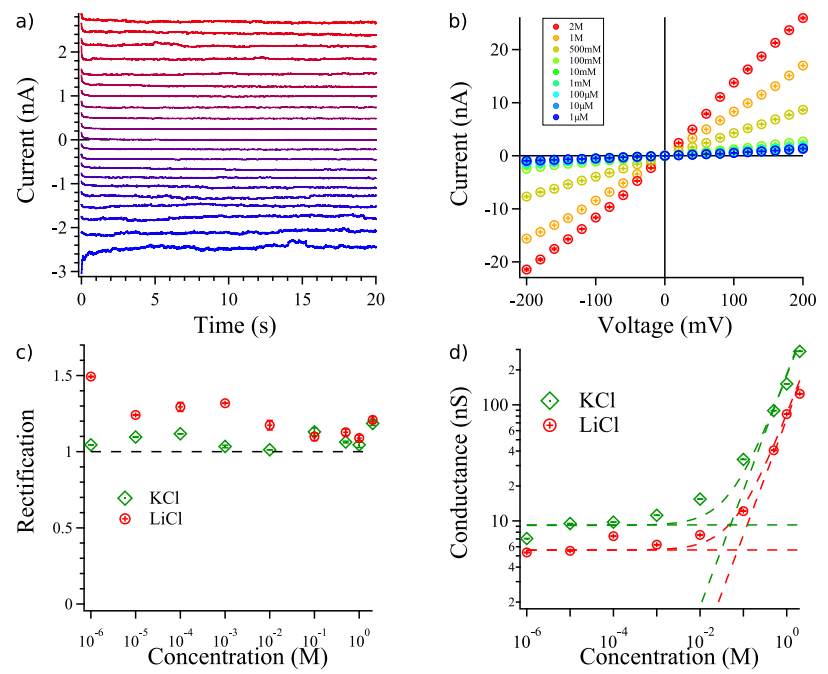

Figure 4: Characterization of a hairy $\mathrm{Si}_{3} \mathrm{~N}_{4}$ nanopore. a) Typical sequence of current measurements used to obtain one current versus voltage (IV) plot a hairy nanopore. Each trace in this graph corresponds to a voltage ranging from $-200 \mathrm{mV}$ (blue) to $200 \mathrm{mV}$ (red) in a $10 \mathrm{mM} \mathrm{LiCl}$ buffer. b) IV plots obtained with $1 \mu \mathrm{M}$ to $2 \mathrm{M} \mathrm{LiCl}$. c) Rectification $|I(+200 \mathrm{mV}) / I(-200 \mathrm{mV})|$ of the same nanopore according to the $\mathrm{KCl}$ and $\mathrm{LiCl}$ concentrations. d) Conductance of the pore at different $\mathrm{KCl}$ and $\mathrm{LiCl}$ concentrations showing the transition between surface conduction at low concentration and ionic conduction at higher concentrations. Solutions were buffered by $5 \mathrm{mM}$ Hepes at $\mathrm{pH}$ 7.4. Experiments are performed with a 31 $\mathrm{nm}$ wide nanopore grafted with PEG-7kDa in a 10 $\mathrm{mM} \mathrm{LiCl}$ buffer.
$200 \mathrm{mV}) \mid$ according to the $\mathrm{LiCl}$ or $\mathrm{KCl}$ concentration (Figure 4b). When we increase the $\mathrm{LiCl}$ concentration from $1 \mu \mathrm{M}$ to $10 \mathrm{mM}$, the rectification decreases from 1.49 to 1.17 . For concentrations larger than $10 \mathrm{mM}$, this rectification remains steady $(1.14 \pm 0.05)$. In presence of $\mathrm{KCl}$, the rectification remains steady $(1.08 \pm 0.06)$ whatever the concentration. Considering the Debye length is larger than $3 \mathrm{~nm}$ if the salt concentration is smaller than $10 \mathrm{mM},{ }^{51}$ the electroosmotic flow could drag the grafted chains along the nanopore. In presence of $\mathrm{KCl}$, the $\mathrm{K}^{+}$cations are linked to the PEG chains, which are too strongly attracted to the negatively charged inner side of the nanopore to be dragged by the electroosmotic flow. In this case, we do not notice any current fluctuation.

Secondly, the average value of each current trace of the Figure 4a is used to plot the current-voltage characteristic curve (I-V curve) of the nanopore (Figure 4b). We observe a linear behavior of the ionic current versus the applied voltage : the ionic conductance through this channel follows the Ohm's law. The IV-curve slope defines the conductance $G$ of the nanopore.

We repeat these steps changing the salt concentration $(1 \mu \mathrm{M}<\mathrm{C}<2 \mathrm{M})$ and nature $(\mathrm{LiCl}$ or $\mathrm{KCl}$ ) to obtain Figure $4 \mathrm{~d}$. This figure shows that the pore conductance $G$ increases with salt concentration according to two regimes. At high concentrations $(C>0.1 \mathrm{M})$, the conductance increases linearly with the salt concentration thus being governed by the bulk ionic transport. At low concentration, the conductance reaches a plateau and is dominated by the movement of counter-ions on the inner surface of the nanopore.

Pore size determination according to its ionic conductance is a frequently discussed matter. ${ }^{34,58,59}$ This conductance can be described by following simplified models. ${ }^{51,60}$ At high ionic concentration, the conductance is governed by the bulk conductivity $K$ : $G_{v o l}=\left(\pi R^{2}\right) / L \times K$ where $R$ is the radius of the pore, $L$ its length. Nevertheless, we must take the access conductance $G_{a c c}=4 R K$ of the nanopore into account. ${ }^{61}$ Considering $G_{\text {ionic }}=1 /\left(1 / G_{v o l}+1 / G_{a c c}\right)$, we write :

$$
G_{\text {ionic }}=\frac{K}{\frac{L}{\pi R^{2}}+\frac{1}{4 R}}
$$


In this regime, the bulk conductivity reads :

$$
K=\left(\mu_{+}+\mu_{-}\right) e^{2} c
$$

where $\mu_{+}$and $\mu_{-}$are the cation $\left(\mu_{K+}=4.76 \times\right.$ $\left.10^{11} \mathrm{~m}^{2} \mathrm{~s}^{-1} \mathrm{~J}^{-1}, \mu_{L i+}=2.50 \times 10^{11} \mathrm{~m}^{2} \mathrm{~s}^{-1} \mathrm{~J}^{-1}\right)$ and the anion $\left(\mu_{C l}=4.94 \times 10^{11} \mathrm{~m}^{2} \mathrm{~s}^{-1} \mathrm{~J}^{-1}\right)$ mobilities respectively, $e$ is the elementary charge and $c$ the ion concentration (in ions per $\mathrm{m}^{3}$ ).

Now, we consider that the thickness $h$ of the grafting is the same on the membrane and in the nanopore. ${ }^{60}$ Then, the apparent radius and length of the nanopore becomes $R^{\prime}=R_{0}-h$ and $L^{\prime}=$ $L_{0}+2 h$ respectively after membrane coating, with $R_{0}$ and $L_{0}$ the radius and length of the pore before grafting. From this, we can deduce a relation between the nanopore dimensions after grafting : $L^{\prime}=L_{0}+2\left(R_{0}-R^{\prime}\right)$. Finally, the equations (1) and (2) become :

$$
G_{\text {ionic }}^{\prime}=\frac{\left(\mu_{+}+\mu_{-}\right) e^{2} c}{\frac{L_{0}+2\left(R_{0}-R^{\prime}\right)}{\pi R^{\prime 2}}+\frac{1}{4 R^{\prime}}}
$$

The fit of the experimental data (Figure 4d) leads to a decrease of the apparent radius of the nanopore after grafting $R_{\mathrm{LiCl}}^{\prime}=9.4 \pm 0.6 \mathrm{~nm}$ in $\mathrm{LiCl}$ buffer and $R_{K C l}^{\prime}=12 \pm 0.2 \mathrm{~nm}$ in $\mathrm{KCl}$ buffer. Thus, we estimate the thickness of the decorated membrane $L_{\mathrm{LiCl}}^{\prime}=32.7 \pm 1.2 \mathrm{~nm}$ and $L_{K C l}^{\prime}=27.5 \pm 0.4 \mathrm{~nm}$ in $\mathrm{LiCl}$ and $\mathrm{KCl}$ buffer respectively. These results show that the grafted chain thickness is $h_{\mathrm{LiCl}}=5.6 \pm 0.6 \mathrm{~nm}$ or $h_{K C l}=3 \pm 0.2 \mathrm{~nm}$ in presence of $\mathrm{LiCl}$ or $\mathrm{KCl}$ respectively.

Now, we discuss the conformation of the polymer chains grafted on the $\mathrm{Si}_{3} \mathrm{~N}_{4}$ surface assuming that there is no overlapping between grafted chains. ${ }^{62-64}$ First, we could consider that these chains have better affinity with water molecules than with the membrane. In this case, they behave according to a good solvent conformation, as it is depicted in Figure 5. In this situation, the polymer chain has a better affinity for $\mathrm{LiCl}$ or $\mathrm{KCl}$ solvent than for itself. The chain size is characterized by the Flory radius $R_{F} \cdot{ }^{65}$ With PEG-7kDa, we calculate $R_{F}=7.3 \mathrm{~nm}$ (Figure 5a). As $R_{F}$ is larger than the grafted chain thickness, we can assume that the PEG-7kDa chains does not follow this regime. Then, we could consider inter-
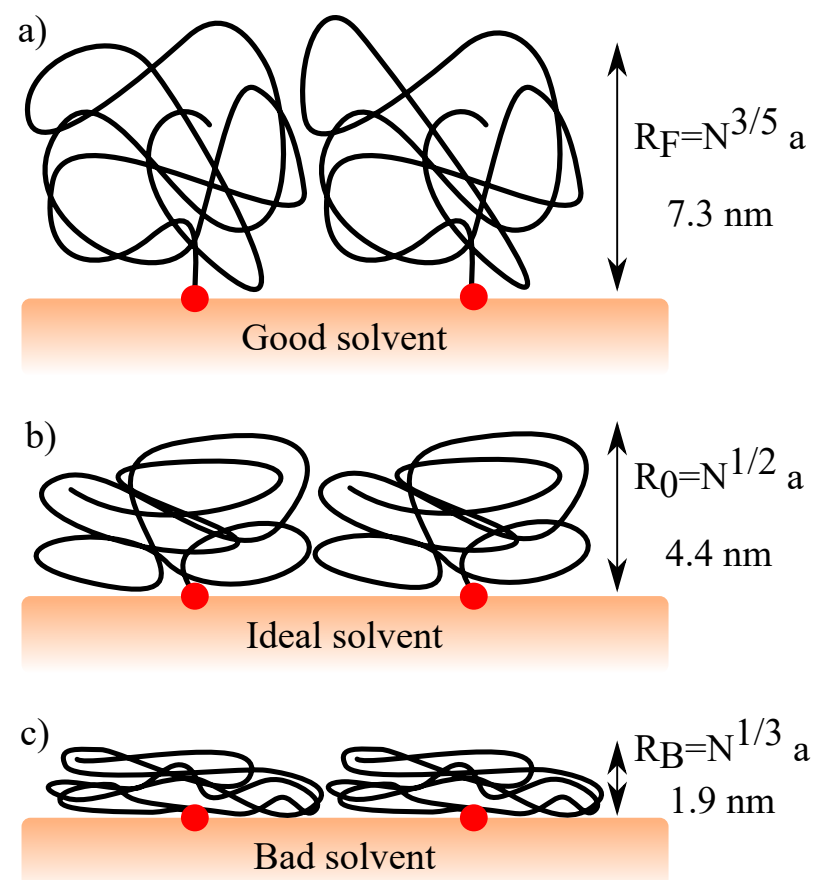

Figure 5: Conformations of polymer chain grafted on $\mathrm{Si}_{3} \mathrm{~N}_{4}$ membrane a) behavior in good solvent regime b) in ideal solvent regime c) in bad solvent regime. $N$ is the number of monomeric units of length $a$. Calculations are perform with PEG$7 \mathrm{kDa}$ chain $(N=159, a=0.35 \mathrm{~nm})$.

actions between polymer chains and the solvent are balanced with the ones with the membrane. In this situation, the polymer chain could be considered as an ideal one characterized by the ideal radius $R_{0}$ (Figure $5 \mathrm{~b}$ ). As $R_{0}=4.4 \mathrm{~nm}$, we could consider that the polymer chains behaves as ideal polymer brush only in presence of $\mathrm{LiCl}$ $\left(h_{\mathrm{LiCl}}>R_{0}\right)$, whereas it is not the case in presence of $\mathrm{KCl}\left(h_{\mathrm{KCl}}<R_{0}\right)$. In the latter case, the PEG chains seem to be collapsed on the membrane in presence of $\mathrm{KCl}$ (Figure 5c). In this situation, the polymer chains are more attracted by the $\mathrm{Si}_{3} \mathrm{~N}_{4}$ surface than by the aqueous phase, and behaves as in bald solvent regime. This behavior could be due to the binding of $K^{+}$cations. ${ }^{66,67}$ Then, the chains adopt a cigar-like shape, as it was previously described in. ${ }^{66}$ The PEG chains behave as positive polyelectrolytes in $\mathrm{KCl}$, which increases their attractions with the negatively charged silanol groups on the membrane surface. ${ }^{68}$ These observations show that the conformation of grafted PEG chains is controlled by the electrolyte: apparent ideal chain in $\mathrm{LiCl}$ and apparent collapsed chain 
in $\mathrm{KCl}$.

At low ionic concentration, the conductance is dominated by surface effects due to the presence of counter-ions on the inner surface of the nanopore.

It reads :

$$
G_{s}=\frac{2 \pi R^{\prime}}{L_{0}} \mu_{+} e \sigma
$$

with $\sigma$ the surface charge of the pore, $\mu_{+}$ the ionic mobility of the positive counterions, considering that the negative charge surface is due to the the dissociation of silanol groups. ${ }^{68}$ At low salt concentrations, we can see on Figure 4 that the pore yields a lower conductance with $\mathrm{LiCl}$ than with $\mathrm{KCl}$ : $G_{s}^{L i C l}=5.4 \pm 0.1 \mathrm{nS}$ and $G_{s}^{\mathrm{KCl}}=9.4 \pm 0.2 \mathrm{nS}$. According to Eq. (4), we calculate the corresponding surface charge : $\sigma_{\mathrm{LiCl}}=46 \pm 6 \mathrm{mC} / \mathrm{m}^{2}$ and $\sigma_{K C l}=33 \pm 3 \mathrm{mC} / \mathrm{m}^{2}$. The surface charge of the grafted nanopore is not dependent on $\mathrm{LiCl}$ or $\mathrm{KCl}$ concentration. These values are in good agreement with the one measured with shorter PEG chain, ${ }^{26}$ but they are smaller than the surface charge measured with uncoated $\mathrm{Si}_{3} \mathrm{~N}_{4}$ channels $\sigma_{\mathrm{SiN}}=60 \mathrm{mC} / \mathrm{m}^{2} .{ }^{50}$ This decrease could be explained by the polymer chain brush covering the inner side of the nanopore. Moreover, this screening effect could be enhanced by the formation of $K^{+} / \mathrm{PEG}$ complexes in presence of $\mathrm{KCl}$ (decrease from 60 to $33 \mathrm{mC} / \mathrm{m}^{2}$ ). ${ }^{66}$

Now, we propose to use this nanopore to characterize interactions between proteins and urea at the nanometer scale. Protein denaturation is usually performed with urea addition. Nevertheless this process is not yet well understood. Two mechanisms are usually described: ${ }^{69}$ the first one is based on the disruption of the structure of water around the protein, which increases the solubility of hydrophobic groups. ${ }^{70}$ These bindings would lead to the entering of urea into the unfolded protein. Thus, urea would behave as a surfactant between the protein and water. ${ }^{71}$ The second mechanism is based on direct interactions between urea and the protein. ${ }^{72}$

In this work, PEG chains act as a model chain to mimic interactions of proteins with urea or water. As the electrical detection coupled with microfluidic integration allows to precise the PEG conformation, we use this method to determine the PEG chain conformation in presence of $\mathrm{LiCl}$
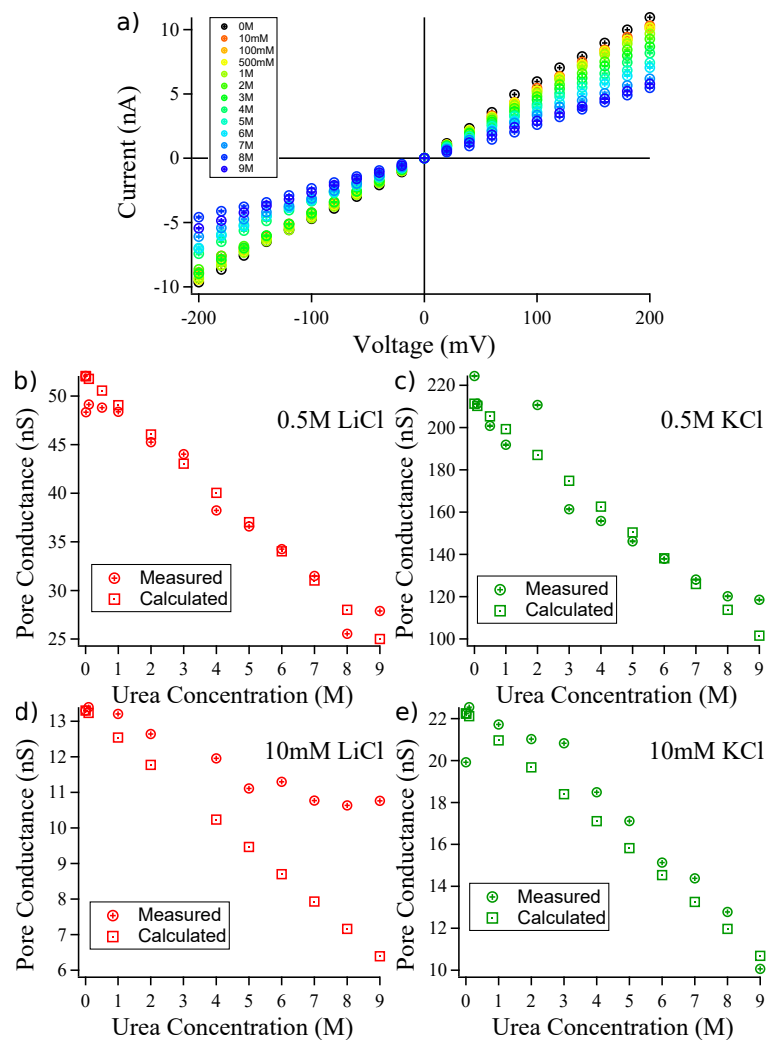

Figure 6: Interactions of urea with polymer brushes in confined medium. a) IV curves obtained with urea concentrations ranging from $10 \mathrm{mM}$ to $9 \mathrm{M}$ at $0.5 \mathrm{M} \mathrm{LiCl}$. Pore conductance (plotted with circle markers) versus urea concentration at b) $0.5 \mathrm{M} \mathrm{LiCl}$, c) $0.5 \mathrm{M} \mathrm{KCl} \mathrm{d)} 10 \mathrm{mM}$ $\mathrm{LiCl} \mathrm{e)} 10 \mathrm{mM} \mathrm{KCl}$. The conductance calculated from bulk conductivity (Eq. 5) is plotted with square markers. All experiments were performed in buffered solution with $5 \mathrm{mM}$ Hepes at $\mathrm{pH}$ 7.5. 
or $\mathrm{KCl}$. Firstly, we consider the high salt concentration where the conductance is governed by the ionic flow. We plot IV-curves in a $0.5 \mathrm{M} \mathrm{LiCl}$ solution by increasing the urea concentration from $0 \mathrm{M}$ to $9 \mathrm{M}$ (Figure 6a and S5). As we do not notice current rectification, we use the slope of each IV-curve to calculate the nanopore conductance. In presence of $0.5 \mathrm{M} \mathrm{LiCl}$, we observe that the nanopore conductance decreases linearly from $52.1 \pm 0.1 \mathrm{nS}$ to $25 \pm 2.3 \mathrm{nS}$ when urea concentration goes from 0 up to $9 \mathrm{M}$ (Figure $7 \mathrm{~b}$ ). As the ionic mobility is inversely proportional to the viscosity, this decrease could be due to the viscosity increase, when we increase the urea concentration. To answer this assumption, we consider a conductance $G_{v}$ calculated from conductivity $K$ of the urea solution :

$$
G_{v}=\frac{K}{K_{b}} G_{b}
$$

with $G_{b}$ and $K_{b}$ the pore conductance and the electrolyte conductivity without urea respectively. This relation, based only on the viscosity variations, is plotted in Figure 6b with square markers. At $0.5 \mathrm{M} \mathrm{LiCl}$, there is a good agreement between conductance measurements, plotted with circle markers, and calculated conductance $G_{v}$. We observe the same behavior at $0.5 \mathrm{M} \mathrm{KCl}$ (Figure $6 \mathrm{c})$. Then, we repeat these measurements at low salt concentration where the conductance is dominated by counter-ions, i.e. charge density on the inner side of the nanopore. We observe the same behavior with $10 \mathrm{mM} \mathrm{KCl}$ (Figure 6e), but the conductance does not decrease as much as expected as the viscosity increases when we add urea in presence of $10 \mathrm{mM} \mathrm{LiCl}$ (Figure 6d).

Then, we normalize the conductance $G$ with the conductance $G_{v}$ calculated above (Eq. 5) to know if the conductance variations are due to viscosity or to geometrical ones :

$$
G_{N}=\frac{G}{G_{v}}
$$

Considering the regime of high ionic concentrations, we observe that the normalized conductance $G_{N}$ is constant $G_{N}=0.99 \pm 0.05$ at $0.5 \mathrm{M} \mathrm{LiCl}$ (Figure 7a). In this regime, the conductance inside the nanopore is mainly governed by the ionic flow. We could neglect the effects due to the surface conductance of the pore. According to Eq. 3, $G_{N}$ is just function of the geometrical dimensions of the nanopore. Then, we define the normalized radius :

$$
R_{N}=\frac{R^{\prime}}{R_{b}}
$$

where $R^{\prime}$ and $R_{b}$ represent the apparent nanopore radius with or without urea respectively, calculated from Eq. 3. In Figure 7c, this normalized radius is steady when the urea concentration increases $R_{N}=0.99 \pm 0.02$. Then, we could suppose that urea has no action on the pore sizes and doesn't affect the conformation of PEG chains at high $\mathrm{LiCl}$ concentrations. We observe the same behavior in presence of $0.5 \mathrm{M} \mathrm{KCl}$ (Figure 7a). In this case, the normalized conductance does not vary $\left(G_{N}=0.96 \pm 0.07\right)$. Then, we plot the normalized radius according to Eq. 7 versus the urea concentration. It is constant $R_{N}=0.98 \pm 0.03$. Then, urea seems to not affect the conformation of grafted PEG chains in presence of $\mathrm{KCl}$, as it was the case with $\mathrm{LiCl}$.

Now, we could consider the regime of low ionic concentrations. If we plot the normalized conductance $G_{N}$, we observe that it increases with urea concentration (Figure 7b). Considering that the nanopore radius is not modified by the increase of urea concentration, this increase could be due to the increase of counter-ion charge according to Eq. 4. Then, we define a normalized surface charge:

$$
\sigma_{N}=\frac{\sigma}{\sigma_{b}}
$$

where $\sigma$ and $\sigma_{b}$ represent the nanopore charge surface with or without urea respectively, calculated from Eq. 4. $\sigma_{N}$ increases with urea concentration until 2.7 times at $9 \mathrm{M}$ urea (Figure $7 \mathrm{~d}$ ). This strong increase could be explained by the high dipole moment of urea $(4.56 \mathrm{D})$, which is 2.5 times higher than the water one $(1.85 \mathrm{D})$. Then, we could suppose that urea could replace water inside the PEG brush and modify strongly the counter-ions distribution inside the nanopore.

Now, we consider the experiments performed with $10 \mathrm{mM} \mathrm{KCl}$. We observe that the normalized conductance of the pore doesn't increase with urea concentration : $G_{N}=1.03 \pm 0.07$ (Figure 7b). Considering that the nanopore conductance is gov- 

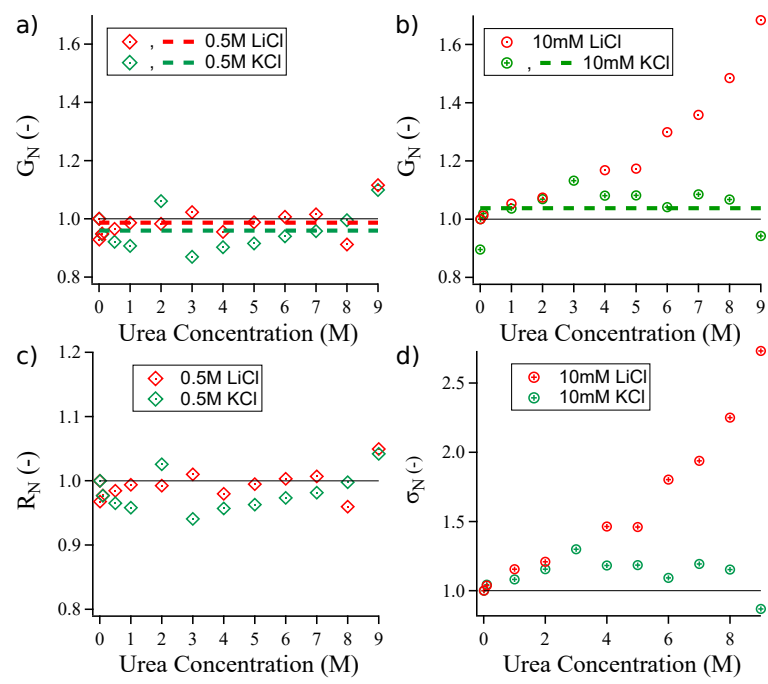

Figure 7: Normalized pore conductance as a function of urea concentration at a) $0.5 \mathrm{M} \mathrm{LiCl}$ and $\mathrm{KCl}$ and b) $10 \mathrm{mM} \mathrm{LiCl}$ and $\mathrm{KCl}$. The dotted lines represents the average values of the conductance. None have been plotted for $10 \mathrm{mM} \mathrm{LiCl}$. The plain black line represents the expected value of $G_{N}=1$. c) Normalized pore radius at $0.5 \mathrm{M} \mathrm{LiCl}$ and $\mathrm{KCl}$ at various urea concentrations. d) Normalized surface charge of the nanopore at $10 \mathrm{mM}$ $\mathrm{LiCl}$ and $\mathrm{KCl}$ at various urea concentrations.

erned by the counter-ions, we plot the normalized charge surface $\sigma_{N}$ in Figure 7d. As $\sigma_{N}$ is steady $\sigma_{N}=1.08 \pm 0.15$, the surface charge seems to not be modified by the addition of urea. Contrary to the observations performed with $\mathrm{LiCl}$, urea molecules seem to not replace the water ones inside the PEG brush in presence of $\mathrm{KCl}$. These observations could be explained by considering the PEG conformation. Previously, we have shown that the PEG chains are strongly attracted to the $\mathrm{Si}_{3} \mathrm{~N}_{4}$ surface in presence of $\mathrm{KCl}$. Then, in that state the polymer brush behaves like in an apparent bad solvent. In this collapsed conformation, we could suppose urea can't go inside the PEG brush to replace water.

We designed and manufactured a cheap microfluidic master from an easy and fast 3D-printerbased protocol for the reversible integration of a solid-state nanopore chip. This device permits to quickly perform a large series of electrical measurements in different environments (nature and concentration of salt, urea) without disassembling the nanopore from the microfluidic sys- tem. Also, our device could be used to rapidly pierce nanopores thanks to the dielectric breakdown method ${ }^{35-37}$ to be then stored for future use. We found that, at low salt concentrations, the conductance is dominated by the movement of counter-ions on the inner surface of the nanopore and that at high concentrations, the conductance is governed by the bulk ionic transport. From electrical measurements we can deduce the conformation of the polymer chains grafted on the nanopore surface. At high ionic concentration, the polymer chains behave as ideal polymer brush or as a cigarlike shape respectively in presence of $\mathrm{LiCl}$ or $\mathrm{KCl}$. For ideal chains, urea molecules can stand in for the water molecules leading to an increase of the apparent charge of the polymer-grafted surface. On the other hand, if the chain is collapsed, the urea molecule cannot enter into the chain and thus don't change its conformation nor its polarisability. This biomimetic system allows to enhance our understanding of interactions between urea and proteins. In our case, the PEG chains in presence of $\mathrm{LiCl}$ are similar to the hydrophilic domains of the protein whereas in presence of $\mathrm{KCl}$ they behave like the hydrophobic domains. This experimental study is in good agreement with previous simulations of protein denaturation with urea. ${ }^{69,72}$ Nevertheless, we did not observe the penetration of urea into the hydrophobic core. ${ }^{72}$

The authors declare no competing financial interest.

Acknowledgement This research was supported by the "DEFI CNRS Instrumentation aux limites" 2016 \& 2017 and by ANR Epsilomics (ANR17-CE09-0044-02). We thank Johanna Canovas, Rasta Ghasemi and Abdelghani Oukhaled for their assistance. This publication is dedicated to Loïc Auvray's memory.

\section{Supporting Information Avail- able}

The Supporting Information is available free of charge on the ACS Publications website at DOI:

Description of a traditional flow-cell; Calculation of the roughness measurement; Flow simulation in microfluidic channels; Current traces 
and fluctuations with hairy nanopore; Conductance measurement in urea;

\section{References}

(1) Lepoitevin, M.; Ma, T.; Bechelany, M.; Janot, J.M.; Balme, S. Functionalization of single solid state nanopores to mimic biological ion channels: A review. Adv. Colloid Interface Sci. 2017, 250, 195-213.

(2) Shi, W.; Friedman, A. K.; Baker, L. A. Nanopore Sensing. Analytical Chemistry 2017, 89, 157188.

(3) Wang, Y.; Zheng, D.; Tan, Q.; Wang, M. X.; $\mathrm{Gu}, \mathrm{L} . \mathrm{Q}$. Nanopore-based detection of circulating microRNAs in lung cancer patients. Nat Nano 2011, $\underline{6}, 668-674$.

(4) Lin, Y.; Ying, Y.-L.; Shi, X.; Liu, S.-C.; Long, Y.T. Direct sensing of cancer biomarkers in clinical samples with a designed nanopore. Chem Commun 2017, 53, 11564-11567.

(5) Coulter, W. H. Means for counting particles suspended in a fluid. 1953.

(6) Larkin, J.; Henley, R. Y.; Muthukumar, M.; Rosenstein, J. K.; Wanunu, M. High-Bandwidth Protein Analysis Using Solid-State Nanopores. Biophysical Journal 2014, 106, 696-704.

(7) Plesa, C.; Cornelissen, L.; Tuijtel, M. W.; Dekker, C. Non-equilibrium folding of individual DNA molecules recaptured up to 1000 times in a solid state nanopore. Nanotechnology 2013, 24, 475101-475108.

(8) Varongchayakul, N.; Huttner, D.; Grinstaff, M. W.; Meller, A. Sensing Native Protein Solution Structures Using a Solid-state Nanopore: Unraveling the States of VEGF. Sci. Rep. 2018, 8, 1017-1025.

(9) Steinbock, L. J.; Otto, O.; Skarstam, D. R.; Jahn, S.; Chimerel, C.; Gornall, J. L.; Keyser, U. F. Probing DNA with micro- and nanocapillaries and optical tweezers. J Phys : Condens Matter 2010, 22, 454113-454120.

(10) Saleh, O. A.; Sohn, L. L. Direct detection of antibody-antigen binding using an on-chip artificial pore. Proc. Natl. Acad. Sci. U. S. A. 2003, 100, 820-824.
(11) Halpern, A. R.; Donavan, K. C.; Penner, R. M.; Corn, R. M. Wafer-scale fabrication of nanofluidic arrays and networks using nanoimprint lithography and lithographically patterned nanowire electrodeposition gold nanowire masters. Anal. Chem. 2012, 84, 5053-5058.

(12) Angeli, E.; Volpe, A.; Fanzio, P.; Repetto, L.; Firpo, G.; Guida, P.; Lo Savio, R.; Wanunu, M.; Valbusa, U. Simultaneous Electro-Optical Tracking for Nanoparticle Recognition and Counting. Nano Letters 2015, 15, 5696-5701.

(13) Siwy, Z.; Fulinski, A. Fabrication of a synthetic nanopore ion pump. Phys. Rev. Lett. 2002, 89, 198103-198106.

(14) Yameen, B.; Ali, M.; Neumann, R.; Ensinger, W.; Knoll, W.; Azzaroni, O. Single conical nanopores displaying $\mathrm{pH}$-tunable rectifying characteristics. manipulating ionic transport with zwitterionic polymer brushes. J. Am. Chem. Soc. 2009, 131, 2070-2071.

(15) Guo, W.; Tian, Y.; Jiang, L. Asymmetric Ion Transport through Ion-Channel-Mimetic SolidState Nanopores. Acc. Chem. Res. 2013, 46, 2834-2846.

(16) Balme, S.; Picaud, F.; Manghi, M.; Palmeri, J.; Bechelany, M.; Cabello-Aguilar, S.; AbouChaaya, A.; Miele, P.; Balanzat, E.; Janot, J. M. Ionic transport through sub-10 nm diameter hydrophobic high-aspect ratio nanopores: experiment, theory and simulation. Sci. Rep. 2015, 5, 10135-10148.

(17) Bacri, L.; Benkhaled, A.; Guegan, P.; Auvray, L. Ionic channel behavior of modified cyclodextrins inserted in lipid membranes. Langmuir 2005, 21, 5842-5846.

(18) Geng, J.; Kim, K.; Zhang, J.; Escalada, A.; Tunuguntla, R.; Comolli, L. R.; Allen, F. I.; Shnyrova, A. V.; Cho, K. R.; Munoz, D.; Wang, Y. M.; Grigoropoulos, C. P.; Ajo-Franklin, C. M.; Frolov, V. A.; Noy, A. Stochastic transport through carbon nanotubes in lipid bilayers and live cell membranes. Nature 2014, 514, 612-615.

(19) Mamad-Hemouch, H.; Ramoul, H.; Abou Taha, M.; Bacri, L.; Huin, C.; Przybylski, C.; Oukhaled, A.; Thiébot, B.; Patriarche, G.; Jarroux, N.; Pelta, J. Biomimetic Nanotubes Based 
on Cyclodextrins for Ion-Channel Applications. Nano Letters 2015, 15, 7748-7754.

(20) Goepfrich, K.; Li, C.-Y.; Ricci, M.; Bhamidimarri, S. P.; Yoo, J.; Gyenes, B.; Ohmann, A.; Winterhalter, M.; Aksimentiev, A.; Keyser, U. F. Large-Conductance Transmembrane Porin Made from DNA Origami. ACS Nano 2016, 10, 82078214.

(21) Bezrukov, S. M.; Vodyanoy, I.; Parsegian, V. A. Counting polymers moving through a single ion channel. Nature 1994, 370, 279-281.

(22) Wang, H.; Ettedgui, J.; Forstater, J.; Robertson, J. W. F.; Reiner, J. E.; Zhang, H.; Chen, S.; Kasianowicz, J. J. Determining the Physical Properties of Molecules with Nanometer-Scale Pores. ACS Sensors 2018, 3, 251-263.

(23) Li, J.; Stein, D.; McMullan, C.; Branton, D.; Aziz, M. J.; Golovchenko, J. a. Ion-beam sculpting at nanometre length scales. Nature 2001, $\underline{412}$, 166-169.

(24) Bacri, L.; Oukhaled, A. G.; Schiedt, B.; Patriarche, G.; Bourhis, E.; Gierak, J.; Pelta, J.; Auvray, L. Dynamics of colloids in single solid-state nanopores. J. Phys. Chem. B 2011, 115, 28902898.

(25) Cressiot, B.; Oukhaled, A.; Patriarche, G.; Pastoriza-Gallego, M.; Betton, J.-M.; Auvray, L.; Muthukumar, M.; Bacri, L.; Pelta, J. Protein Transport through a Narrow Solid-State Nanopore at High Voltage: Experiments and Theory. ACS Nano 2012, 6, 6236-6243.

(26) Giamblanco, N.; Coglitore, D.; Janot, J. M.; Coulon, P. E.; Charlot, B.; Balme, S. Detection of protein aggregate morphology through single antifouling nanopore. Sensors and Actuators, B: Chemical 2018, 260, 736-745.

(27) Oukhaled, A.; Cressiot, B.; Bacri, L.; PastorizaGallego, M.; Betton, J.-M.; Bourhis, E.; Jede, R.; Gierak, J.; Auvray, L.; Pelta, J. Dynamics of Completely Unfolded and Native Proteins through Solid-State Nanopores as a Function of Electric Driving Force. ACS Nano 2011, 5, 36283638 .

(28) Waduge, P.; Hu, R.; Bandarkar, P.; Yamazaki, H.; Cressiot, B.; Zhao, Q.; Whitford, P. C.; Wanunu, M. Nanopore-Based Measurements of
Protein Size, Fluctuations, and Conformational Changes. ACS Nano 2017, 11, 5706-5716.

(29) Larkin, J.; Henley, R.; Bell, D. C.; CohenKarni, T.; Rosenstein, J. K.; Wanunu, M. Slow DNA Transport through Nanopores in Hafnium Oxide Membranes. ACS Nano 2013, 7, 1012110128.

(30) Storm, A. J.; Chen, J. H.; Ling, X. S.; Zandbergen, H. W.; Dekker, C. Fabrication of solid-state nanopores with single-nanometre precision. Nat. Mater. 2003, 2, 537-540.

(31) Baaken, G.; Halimeh, I.; Bacri, L.; Pelta, J.; Oukhaled, A.; Behrends, J. C. High-Resolution Size-Discrimination of Single Nonionic Synthetic Polymers with a Highly Charged Biological Nanopore. ACS Nano 2015, 9, 6443-6449.

(32) Peraro, M. D.; van der Goot, F. G. Pore-forming toxins: ancient, but never really out of fashion. Nat. Rev. Microbiol. 2016, 14, 77-92.

(33) Wanunu, M.; Dadosh, T.; Ray, V.; Jin, J.; Mcreynolds, L.; Drndic, M. microRNAs using thin nanopore sensors. Nature Nanotechnology 2010, 5, 807-814.

(34) Lee, C.; Joly, L.; Siria, A.; Biance, A.-L.; Fulcrand, R.; Bocquet, L. Large apparent electric size of solid-state nanopores due to spatially extended surface conduction. Nano Lett. 2012, 12, 40374044.

(35) Kwok, H.; Briggs, K.; Tabard-Cossa, V. Nanopore fabrication by controlled dielectric breakdown. PLoS One 2014, 9, e92880-e92885.

(36) Feng, J.; Liu, K.; Graf, M.; Dumcenco, D.; Kis, A.; Di Ventra, M.; Radenovic, A. Observation of ionic Coulomb blockade in nanopores. Nat. Mater. 2016, 15, 850-855.

(37) Lin, Y.; Shi, X.; Liu, S.-C.; Ying, Y.-L.; Li, Q.; Gao, R.; Fathi, F.; Long, Y.-T.; Tian, H. Characterization of DNA duplex unzipping through a sub-2 nm solid-state nanopore. Chem. Commun. 2017, 53, 3539-3542.

(38) Niedzwiecki, D. J.; Grazul, J.; Movileanu, L. Single-molecule observation of protein adsorption onto an inorganic surface. Journal of the American Chemical Society 2010, 132, 1081610822. 
(39) Wanunu, M.; Meller, A. Chemically modified solid-state nanopores. Nano Lett. 2007, 7, 15801585 .

(40) Kowalczyk, S. W.; Kapinos, L.; Blosser, T. R.; Magalhaes, T.; van Nies, P.; Lim, R. Y. H.; Dekker, C. Single-molecule transport across an individual biomimetic nuclear pore complex. Nat. Nanotechnol. 2011, 6, 433-438.

(41) Ketterer, P.; Ananth, A. N.; Laman Trip, D. S.; Mishra, A.; Bertosin, E.; Ganji, M.; van der Torre, J.; Onck, P.; Dietz, H.; Dekker, C. DNA origami scaffold for studying intrinsically disordered proteins of the nuclear pore complex. Nat Commun 2018, 9, 902-908.

(42) Roman, J.; Jarroux, N.; Patriarche, G.; Français, O.; Pelta, J.; Le Pioufle, B.; Bacri, L. Functionalized Solid-State Nanopore Integrated in a Reusable Microfluidic Device for a Better Stability and Nanoparticle Detection. ACS Applied Materials \& Interfaces 2017, 41634-41640.

(43) Yin, B.; Xie, W.; Liang, L.; Deng, Y.; He, S.; He, F.; Zhou, D.; Tlili, C.; Wang, D. Covalent Modification of Silicon Nitride Nanopore by Amphoteric Polylysine for Short DNA Detection. ACS Omega 2017, 2, 7127-7135.

(44) Tahvildari, R.; Beamish, E.; Tabard-Cossa, V.; Godin, M. Integrating nanopore sensors within microfluidic channel arrays using controlled breakdown. Lab on a Chip 2015, 15, 1407-1411.

(45) Tahvildari, R.; Beamish, E.; Briggs, K.; ChagnonLessard, S.; Sohi, A. N.; Han, S.; Watts, B.; Tabard-Cossa, V.; Godin, M. Manipulating Electrical and Fluidic Access in Integrated NanoporeMicrofluidic Arrays Using Microvalves. Small 2016, 13, 1602601-1602607.

(46) Duffy, D. C.; McDonald, J. C.; Schueller, O. J. A.; Whitesides, G. M. Rapid prototyping of microfluidic systems in poly(dimethylsiloxane). Analytical Chemistry 1998, 70, 4974-4984.

(47) Mcdonald, J. C.; Chabinyc, M. L.; Metallo, S. J.; Anderson, J. R.; Stroock, A. D.; Whitesides, G. M. Prototyping of Microfluidic Devices in Poly ( dimethylsiloxane ) Using Solid-Object Printing the fabrication of microfluidic devices in poly ( dimethyl-. Analytical chemistry 2002, 74, 1537-1545.
(48) Macdonald, N. P.; Cabot, J. M.; Smejkal, P.; Guijt, R. M.; Paull, B.; Breadmore, M. C. Comparing Microfluidic Performance of ThreeDimensional (3D) Printing Platforms. Analytical Chemistry 2017, 89, 3858-3866.

(49) Peleg, O.; Tagliazucchi, M.; Kroeger, M.; Rabin, Y.; Szleifer, I. Morphology Control of Hairy Nanopores. ACS Nano 2011, 5, 4737-4747.

(50) Stein, D.; Kruithof, M.; Dekker, C. Surfacecharge-governed ion transport in nanofluidic channels. Phys. Rev. Lett. 2004, 93, 035901035904 .

(51) Schoch, R. B.; Han, J.; Renaud, P. Transport phenomena in nanofluidics. Reviews of Modern Physics 2008, 80, 839-883.

(52) Myers, J. K.; Pace, C. N.; Scholtz, J. M. Denaturant $m$ values and heat capacity changes: Relation to changes in accessible surface areas of protein unfolding. Protein Science 1995, 4, 2138-2148.

(53) Vanzi, F.; Madan, B.; Sharp, K. Effect of the Protein Denaturants Urea and Guanidinium on Water Structure: A Structural and Thermodynamic Study. Journal of the American Chemical Society 1998, 120, 10748-10753.

(54) Bennion, B. J.; Daggett, V. The molecular basis for the chemical denaturation of proteins by urea. Proceedings of the National Academy of Sciences 2003, 100, 5142-5147.

(55) Yang, H.; Zhang, G.; Hong, X.; Zhu, Y. Silylation of mesoporous silica MCM-41 with the mixture of $\mathrm{Cl}(\mathrm{CH} 2) 3 \mathrm{SiCl} 3$ and $\mathrm{CH} 3 \mathrm{SiCl} 3$ : combination of adjustable grafting density and improved hydrothermal stability. Microporous and

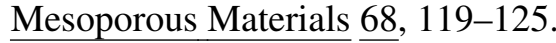

(56) Tang, C. K.; Vaze, A.; Rusling, J. F. Automated 3D-printed unibody immunoarray for chemiluminescence detection of cancer biomarker proteins. Lab Chip 2017, 17, 484-489.

(57) Milner, S. T. Polymer Brushes. Science 1991, 251, 905-914.

(58) Ma, Y.; Guo, J.; Jia, L.; Xie, Y. Entrance Effects Induced Rectified Ionic Transport in a Nanopore/Channel. ACS Sensors 2018, 3, 167173. 
(59) Wen, C.; Zhang, Z.; Zhang, S. L. Physical Model for Rapid and Accurate Determination of Nanopore Size via Conductance Measurement. ACS Sensors 2017, 2, 1523-1530.

(60) Frament, C. M.; Bandara, N.; Dwyer, J. R. Nanopore Surface Coating Delivers Nanopore Size and Shape through Conductance-Based Sizing. ACS Applied Materials \& InterfacesApplied Materials \& Interfaces 2013, 5, 9330-9337.

(61) Hall, J. E. Access resistance of a small circular pore. The Journal of General Physiology 1975, $66,531-532$.

(62) Alexander, S. Adsorption of chain molecules with a polar head a scaling description. 1977, 38, 983987.

(63) Gennes, P. G. D. Conformations of Polymers Attached to an Interface. Macromolecules 1980, 13, 1069-1075.

(64) Auroy, P.; Auvray, L.; Léger, L. Characterization of the brush regime for grafted polymer layers at the solid-liquid interface. Physical Review Letters 1991, 66, 719-722.

(65) De Gennes, P. G. Scaling Concepts in Polymer Physics; Cornell University Press, Ithaca, NY, 1979.

(66) Reiner, J. E.; Kasianowicz, J. J.; Nablo, B. J.; Robertson, J. W. F. Theory for polymer analysis using nanopore-based single-molecule mass spectrometry. Proc. Natl. Acad. Sci. U. S. A. 2010, 107, 12080-12085.

(67) Breton, M. F.; Discala, F.; Bacri, L.; Foster, D.; Pelta, J.; Oukhaled, A. Exploration of Neutral Versus Polyelectrolyte Behavior of Poly(ethylene glycol)s in Alkali Ion Solutions using SingleNanopore Recording. The Journal of Physical Chemistry Letters 2013, 4, 2202-2208.

(68) Behrens, S. H.; Grier, D. G. The charge of glass and silica surfaces. Journal of Chemical Physics 2001, 115, 6716-6721.

(69) Xia, Z.; Das, P.; Shakhnovich, E. I.; Zhou, R. Collapse of unfolded proteins in a mixture of denaturants. Journal of the American Chemical Society 2012, 134, 18266-18274.
(70) Hammes, G. G.; Schimmel, P. R. An Investigation of Water-Urea and Water-Urea-Polyethylene Glycol Interactions. J. Am. Chem. Soc. 1967, 89, 442-446.

(71) Zangi, R.; Zhou, R.; Berne, B. J. Urea's action on hydrophobic interactions. Journal of the American Chemical Society 2009, 131, 15351541.

(72) Hua, L.; Zhou, R.; Thirumalai, D.; Berne, B. J. Urea denaturation by stronger dispersion interactions with proteins than water implies a 2-stage unfolding. Proceedings of the National Academy of Sciences 2008, 105, 16928-16933. 
Graphical TOC Entry

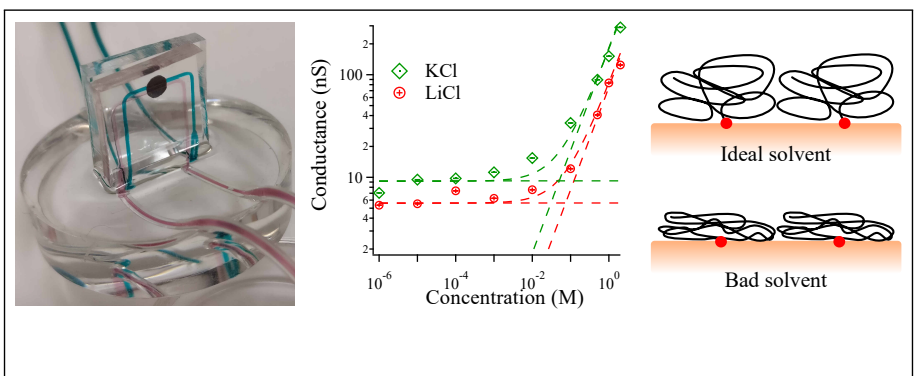

